\title{
Comparación entre dos técnicas de medición del gasto cardíaco mediante ecocardiografía Doppler en pediatría
}

\author{
Guardabassi DS. ${ }^{1,2}$, Fiorito P. ${ }^{2}$, Yanco N. ${ }^{2}$, Cohen L. ${ }^{2}$ \\ 1 Hospital del Niño de San Justo, San Justo, Argentina. \\ 2 Hospital Ramos Mejía, Buenos Aires, Argentina.
}

Introducción: La ecocardiografía transtorácica constituye una herramienta útil para el monitoreo hemodinámico no invasivo en cirugía pediátrica de alta complejidad. Sin embargo, realizar mediciones del gasto cardíaco mediante un examen transtorácico apical resulta técnicamente difícil en el período intraoperatorio. Un abordaje desde la cabecera del paciente, a nivel supraesternal, emerge como alternativa para superar dicha limitante. El objetivo de la presente investigación es comparar ambas mediciones a nivel basal y luego de cambios hemodinámicos (anestesia caudal) en un grupo de pacientes.

Materiales y Métodos: Este estudio descriptivo incluyó a 8 pacientes de 1-6 años de edad sometidos a cirugía de abdomen inferior. En todos los casos estudiados se administró un bloqueo caudal con $1 \mathrm{~mL} / \mathrm{kg}$ de bupivacaína 0,25\% bajo anestesia general. Inmediatamente antes y 10 minutos después de la realización del mismo, se midió mediante ecocardiografía doppler la integral velocidad-tiempo (IVT) a nivel del tracto de salida del ventrículo izquierdo (TSVI) en visión apical 5 cámaras (5C) y la IVT de aorta descendente (AoD) en visión supraesternal (SE). Todas las mediciones fueron realizadas por un mismo operador; se efectuaron y promediaron 3 mediciones en ambas vistas para cada intervalo para su análisis posterior. Los cálculos de gasto cardíaco (GC) fueron realizados mediante la fórmula GC = IVT x área (A), obteniéndose la misma a partir de la medición en vista paraesternal eje largo para el TSVI, y a partir de un normograma según edad para el área de AoD.

Para el análisis estadístico, se utilizaron los análisis de coeficiente de correlación de Pearson y regresión lineal (programa STATA). Un valor de $\mathrm{p}<0,05$ fue considerado como estadísticamente significativo.

Resultados: Los hallazgos muestran una correlación significativa para las mediciones de IVT realizadas a nivel del TSVI y la AoD pre bloqueo caudal: $\mathrm{r}=0,92$ (IC $95 \%$ : 0,89-0,95; $\mathrm{p}<0,001 ; \mathrm{r} 2=0,85$ ) y post bloqueo: $\mathrm{r}=0,78$ (IC 95\%: 0,72-0,81; $\mathrm{p}<0,001 ; \mathrm{r} 2=0,65)$. Dicha correlación se obtuvo también para las mediciones de GC en ambas vistas e intervalos $(\mathrm{r}=0,89$ y 0,$81 ; \mathrm{p}<0,001)$, respectivamente.

Conclusiones: Nuestros resultados muestran una buena correlación entre las mediciones de IVT y GC mediante ecocardiografía en 5C y SE; dicho hallazgo se mantuvo ante un evento de variación hemodinámica (anestesia caudal). Estos resultados sugieren que la medición de IVT a nivel SE puede constituir una herramienta de monitoreo hemodinámico no invasivo, de fácil acceso en la cabecera del paciente, que podría incorporarse a los protocolos de terapia dirigida por objetivos en cirugía de alta complejidad.

https://doi.org/10.25237/congresoclasa2019.33 